\title{
Functionalized Nano Diamonds: Improving Biomedical Features Using Rare-Earth Elements
}

\author{
Ellen Cristine Giese* \\ Center for Mineral Technology, Brazil
}

*Corresponding author: Ellen Cristine Giese, Center for Mineral Technology, CETEM, Av Pedro Calmon 900, CEP 21941 908, Rio de Janeiro, Brazil

\section{ARTICLE INFO}

Received: 豐 November 08, 2019

Published: November 18, 2019

Citation: Ellen Cristine Giese.

Functionalized Nano Diamonds: Improving Biomedical Features Using Rare-Earth Elements. Biomed J Sci \& Tech Res 22(5)2019. BJSTR. MS.ID.003828.

\section{ABSTRACT}

Abbreviations: REE: Rare-Earth Elements; MR: Magnetic Resonance; LA: Lanthanum; CE: Cerium; PR: Praseodymium; ND: neodymium; PM: Promethium; SM: Samarium; EU: Europium; GD: Gadolinium; TB: Terbium; DY: Dysprosium; HO: Holmium; ER: Erbium; TM: Thulium; YB: Ytterbium; LU: Lutetium

\section{Opinion}

Nano diamonds (NDs) or diamond nanoparticles consist of single-crystal diamonds of 4-5nm that presents $\mathrm{sp} 3$ carbon as the main component with high physical and chemical properties. NDs exhibit tunable surface, excellent biocompatibility and large surface area for conjugation of molecules like drugs and genes for their intracellular and extracellular delivery [1]. Surface functionalization of NDs is necessary to improve the circulation half-life and avoid their faster elimination via opsonization by phagocytes in the human body which makes them unsuitable for sustained drug delivery [2]. Functionalized NDs have emerged as a good solution to improve the NDs performance for delayed drug delivery, image probes, or implant coatings in biological systems [1]. In a recent paper, a team of Zhengzhou researchers fabricated covalently functionalizing NDs with rare-earth elements ( $\mathrm{REE}, \mathrm{Eu}^{3+}$, and $\mathrm{Gd}^{3+}$ ) 2-thenoyltrifluoroacetone (TTA) complexes to obtain nanoparticles with simultaneous fluorescence/MR imaging and drug-delivery capabilities [3]. The in vitro/in vivo assays demonstrated the potential of ND-TTA: Eu3+, Gd3+ for optical and magnetic resonance (MR) imaging as also a good drug storage capability that reaches $375 \mu \mathrm{g} / \mathrm{mg}$ towards anticancer drug doxorubicin.

In the last years, NDs have been considered as promising drug carriers to deliver a broad range of therapeutics due to their advantageous features including chemical stability, good biocompatibility, functionalization versatility, unique surface electrostatics, and the large surface specific area, etc. [4-6]. Many of chromophores have been combined noncovalently onto the ND surface, e.g., cytochrome c and fluorescein isothiocyanate. But precise imaging and long-time tracking have been harmed by the inevitable autofluorescence, photo-blinking, and photo-bleaching properties from organic compounds [3]. REE are a group of metals comprised of fourteen lanthanide elements [lanthanum (La), cerium (Ce), praseodymium (Pr), neodymium (Nd), promethium $(\mathrm{Pm})$, samarium $(\mathrm{Sm})$, europium $(\mathrm{Eu})$, gadolinium (Gd), terbium (Tb), dysprosium (Dy), holmium (Ho), erbium (Er), thulium (Tm), ytterbium (Yb), lutetium (Lu)], yttrium (Y) and scandium (Sc) [7]. These elements presenting unique physical and chemical properties have rendered them indispensable for a growing number of hightech technologies [8-9].

REE has been used in many health and medical applications as possess good optical properties. These elements have been applied to tomography scans, magnetic resonance images, positron emission tomography imaging and X-rays [10]. Nowadays, REE has also been considered on the anti-cancer treatment because of its therapeutic radioisotopes, especially as agents in 
radioimmunotherapy and photodynamic therapy [11]. The use of REE into health and medical applications is now well established. The NDs-based multi-functional hybrid rare-earth complex can emit bright red luminescence which has long lifetime reaching $0.68 \mathrm{~ms}$ much longer than that of auto-fluorescence from biotissues. Furthermore, these ND-TTA: $\mathrm{Eu}^{3+}, \mathrm{Gd}^{3+}$ composites [3] are low-toxic and may provide a promising nanoplatform for optical/ MR multimodal imaging. Biomedicine requires powerful imaging techniques of the single-molecule scale to the whole organism, either for fundamental science or diagnosis as well as powerful drug carriers; and rare earth-functionalized nano diamonds showed are promising for dual-modal imaging and drug delivery biological applications. This discovery will be fundamental to the development of new technologies to be used in medicine.

\section{Acknowledgement}

None.

\section{Conflict of Interest}

No conflict of interest.

\section{References}

1. Chauhan S, Jain NG, Negaich U (2019) Nanodiamonds with powerful ability for drug delivery and biomedical applications: Recent updates on in vivo study and patents. Journal of Pharmaceutical Analysis, in press, journal pre-proof, on-line.

\section{ISSN: 2574-1241}

DOI: $10.26717 /$ BJSTR.2019.22.003828

Ellen Cristine Giese. Biomed J Sci \& Tech Res

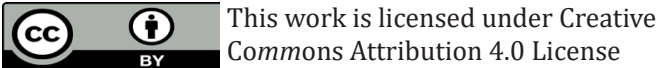

Submission Link: https://biomedres.us/submit-manuscript.php
2. Tisler J, Balasubramanian G, Naydenov B, Kolesov R, Grotz B, et al. (2009) Fluorescence and spin properties of defects in single digit nanodiamonds, ACS Nano 3(7): 1959-1965.

3. Qin SR, Zhao Q, Cheng ZG, Zhang DX, Zhang KK, et al. (2019) Rare earth-functionalized nano diamonds for dual-modal imaging and drug delivery. Diamond and Related Materials 91: 173-182.

4. Mochalin VN, Shenderova O, Ho D, Gogotsi Y (2011) The properties and applications of nanodiamonds. Nature Nanotechnology 7: 11-23.

5. Moore L, Yang J, Lan TTH, Osawa E, Lee DK, et al. (2016) Biocompatibility assessment of detonation nanodiamond in nonhuman primates and rats using histological, hematologic, and urine analysis. ACS Nano 10(8): 7385-7400.

6. Krueger A, Lang D (2012) Functionality is key: recent progress in the surface modification of nanodiamond. Advanced Functional Materials 22(5): 890-906

7. Kumari A, Panda R, Jha MK, Kumar JR, Lee JY (2015) Process development to recover rare earth metals from monazite mineral: A review. Minerals Engineering 79: 102-115.

8. Riba JR, López Torres C, Romeral L, Garcia A (2016) Rare-earth-free propulsion motors for electric vehicles: A technology review. Renewable and Sustainable Energy Reviews 57: 367-379.

9. Cui J, Kramer M, Zhou L, Liu F, Sellmyer D (2018) Current progress and future challenges in rare-earth-free permanent magnets. Acta Materialia 158: 118-137.

10. Giese EC (2019) Rare Earth Elements: therapeutic and diagnostic applications in modern medicine. Clinical and Medical Reports 2(1): 1-2.

11. Kostova I (2005) Lanthanides as Anticancer Agents. Current Medicinal Chemistry - Anti-Cancer Agents 5(6): 591-602.

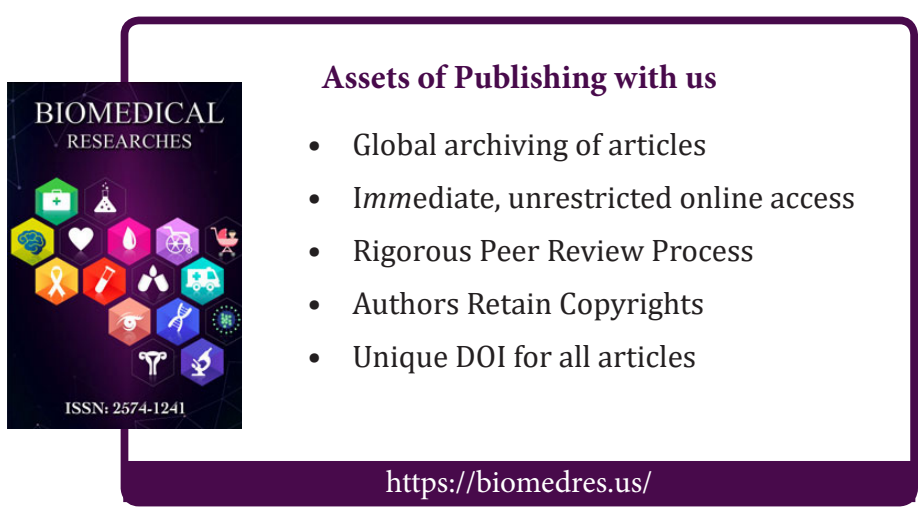

